\title{
DETERMINAÇÃO DE PROPRIEDADES TÉRMICAS DE ALIMENTOS LÍQUIDOS POR MEIO DA TÉCNICA DE SONDA LINEAR DE AQUECIMENTO
}

\author{
M. M. SPESSOTTO ${ }^{1}$, J. A. W. GUT ${ }^{1,2}$ \\ ${ }^{1}$ Universidade de São Paulo, Escola Politécnica, Dep.de Engenharia Química \\ ${ }^{2}$ Universidade de São Paulo, FoRC - Centro de Pesquisa em Alimentos \\ E-mail para contato: jorgewgut@usp.br
}

\begin{abstract}
RESUMO - O objetivo do projeto foi determinar as propriedades térmicas (condutividade térmica, difusividade térmica e calor específico) de alguns alimentos líquidos relevantes para processos térmicos contínuos através do método da sonda linear de aquecimento com imobilização do meio com ágar em diferentes temperaturas. O método foi testado e calibrado com água destilada e aplicado para: suco de laranja, polpa de manga e suco de maçã. Resultados adequados foram obtidos entre $10{ }^{\circ} \mathrm{C}$ e $60{ }^{\circ} \mathrm{C}$, mas o método não pode ser aplicado em temperaturas superiores devido aos efeitos convectivos pronunciados.
\end{abstract}

\section{INTRODUÇÃO}

Em processos térmicos contínuos, o conhecimento sobre as propriedades térmicas dos alimentos processados é fundamental para a realização de projetos de equipamentos industriais, como trocadores de calor. Na indústria alimentícia, esses dados são importantes para a determinação, por exemplo, da penetração de calor em alimentos embalados, para assegurar a segurança alimentar.

A técnica da sonda linear de aquecimento é um método transiente bastante utilizado para medir propriedades térmicas de alimentos devido a sua praticidade, medição rápida, baixo custo, pequena quantidade de amostra necessária e pelo fato de não depender da geometria da amostra, quando há uma grande quantidade de amostra. A medição é baseada no modelo de um sistema ideal composto por uma fonte linear de aquecimento infinita de massa desprezível, imerso num meio sólido infinito, aquecendo esse meio com uma taxa de calor constante (Rahman, 2009). O método foi desenvolvido e revisado por vários pesquisadores, como Stahlhane e Pyk (1933), Van der Held e Van Drunen (1949), Hooper e Lepper (1950), Sweat e Haugh (1974) e Murakami et al. (1996a,b).

Os detalhes da teoria básica sobre o uso da sonda linear de aquecimento foram discutidos por Hooper e Leper (1950) e Nix et al. (1967). A adição de ágar a meios líquidos aquosos é necessária para gelificação para evitar efeitos convectivos (escoamento). Os principais fatores que podem interferir nesse método são a quantidade de ágar adicionado, a duração das análises, a distância entre a sonda e a parede do recipiente, a corrente aplicada e a distância entre as agulhas. $\mathrm{O}$ cuidado com as agulhas do sensor é indispensável para garantir a 


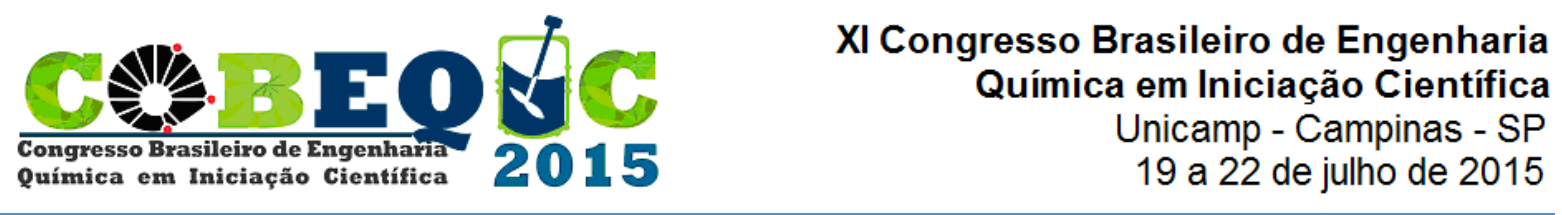

qualidade do método, pois um leve dobramento pode gerar erros consideráveis (Rahman, 2009).

O objetivo desse projeto é estudar a determinação de condutividade térmica, difusividade térmica e calor específico pelo método de sonda linear de aquecimento com imobilização por ágar em suco de laranja, polpa de manga e suco de maçã, verificando o efeito da temperatura.

\section{MATERIAL E MÉTODOS}

Suco de laranjas da variedade Pera foi extraído usando a extratora Fresh (FMC, EUA) segundo Tribess (2003). Polpa de manga da variedade Palmer foi obtida descascando as frutas, retirando o caroço e processando em despolpadeira segundo Sugai (2007). Suco de maçãs da variedade Fuji foi obtido com centrífuga convencional. Para cada suco foram feitos três ensaios de determinação de propriedades, porém os dados não foram tratados como triplicatas pois as temperaturas médias registradas pelo sensor diferem da desejada devido ao aquecimento do meio pela sonda.

As amostras foram acondicionadas em béqueres de $100 \mathrm{~mL}$ e adicionou-se $1,0 \% \mathrm{em}$ massa de ágar (Agar-Agar em pó Type 900). Pesagens foram realizadas em balança semianalítica. Os béqueres utilizados foram selecionados de forma a atender às exigências do KD2 Pro Thermal Properties Analyzer (Decagon Devices, EUA), devendo ter altura suficiente para inserir toda a agulha do sensor e no mínimo $1,5 \mathrm{~cm}$ de raio de distância entre o sensor e a parede do recipiente.

A homogeneização da amostra foi feita com o auxílio de um bastão de vidro. Em seguida a amostra foi aquecida até $65{ }^{\circ} \mathrm{C}$ para gelificação e depois resfriada até $10{ }^{\circ} \mathrm{C}$, temperatura na qual iniciam-se as análises térmicas. A temperatura da amostra foi mantida com o auxílio de um banho ultratermostatizado MA-184 (Marconi, Brasil). A temperatura máxima atingida foi de $60{ }^{\circ} \mathrm{C}$, pois acima dela perde-se o gel. As análises são feitas aproximadamente a cada $10^{\circ} \mathrm{C}$ elevados.

O equipamento utilizado para as análises foi o KD2 Pro Thermal Properties Analyzer (Decagon Devices, EUA) com o sensor de duas agulhas SH-1. O sensor possui a capacidade de medir a condutividade térmica na faixa de 0,02 a $2,00 \mathrm{~W} \mathrm{~K}^{-1} \mathrm{~m}^{-1}$ com precisão de $\pm 5 \%$ de 0,2 a $2,0 \mathrm{~W} \mathrm{~K}^{-1} \mathrm{~m}^{-1}$ e de $\pm 0,01 \mathrm{~W} \mathrm{~K}^{-1} \mathrm{~m}^{-1}$ de 0,02 a $0,2 \mathrm{~W} \mathrm{~K}^{-1} \mathrm{~m}^{-1}$. A precisão na determinação da difusividade é de $\pm 5 \%$ para condutividades acima de $0,1 \mathrm{~W} \mathrm{~K}^{-1} \mathrm{~m}^{-1}$ e a precisão para calor específico volumétrico é de $\pm 7 \%$ para condutividades acima de $0,1 \mathrm{~W} \mathrm{~K}^{-1}$ $\mathrm{m}^{-1}$ (Decagon Devices, 2006).

\section{RESULTADOS E DISCUSSÃO}

A fim de determinar se o método utilizado foi adequado, foram realizados experimentos com água destilada imobilizada com ágar (1,0\% em massa). Os dados obtidos foram comparados com valores da literatura, segundo Lide e Kehiaian (1994). Em temperaturas elevadas os resultados apresentaram variações significativas. Este comportamento deve-se ao fato de que acima dessa temperatura os efeitos convectivos começam a se tornar relevantes, 
fator que interfere na leitura, como foi observado experimentalmente durante a realização desse projeto.

Os valores obtidos experimentalmente diferem ligeiramente do esperado. Essa diferença pode ser causada pela adição do ágar, pela calibração do equipamento ou pelo erro de medição. Consequentemente, foram adotados fatores de correção $f_{k}$, para a condutividade, e $f_{\alpha}$, para a difusividade, conforme sugerido por Rahman (2009). Esses fatores, apresentados abaixo, são obtidos pela razão entre os dados experimentais e os dados da literatura:

$$
f_{k}=\frac{k_{\text {literatura }}}{k_{\text {experimental }}}=0,981 \pm 0,006 \quad f_{\alpha}=\frac{\alpha_{\text {literatura }}}{\alpha_{\text {experimental }}}=0,942 \pm 0,005
$$

Estes fatores foram aplicados para correção dos resultados obtidos com os alimentos testados. Foram utilizadas três amostras do suco de laranja do mesmo lote, que foram imobilizadas com ágar (1,0 \%). Essas amostras foram submetidas a uma faixa de temperatura de 10 a $50{ }^{\circ} \mathrm{C}$ e os dados corrigidos encontram-se na Figura 1.

Figura 1 - Gráficos de condutividade térmica (A), difusividade térmica (B) e calor específico volumétrico $(\mathrm{C})$ de suco de laranja.

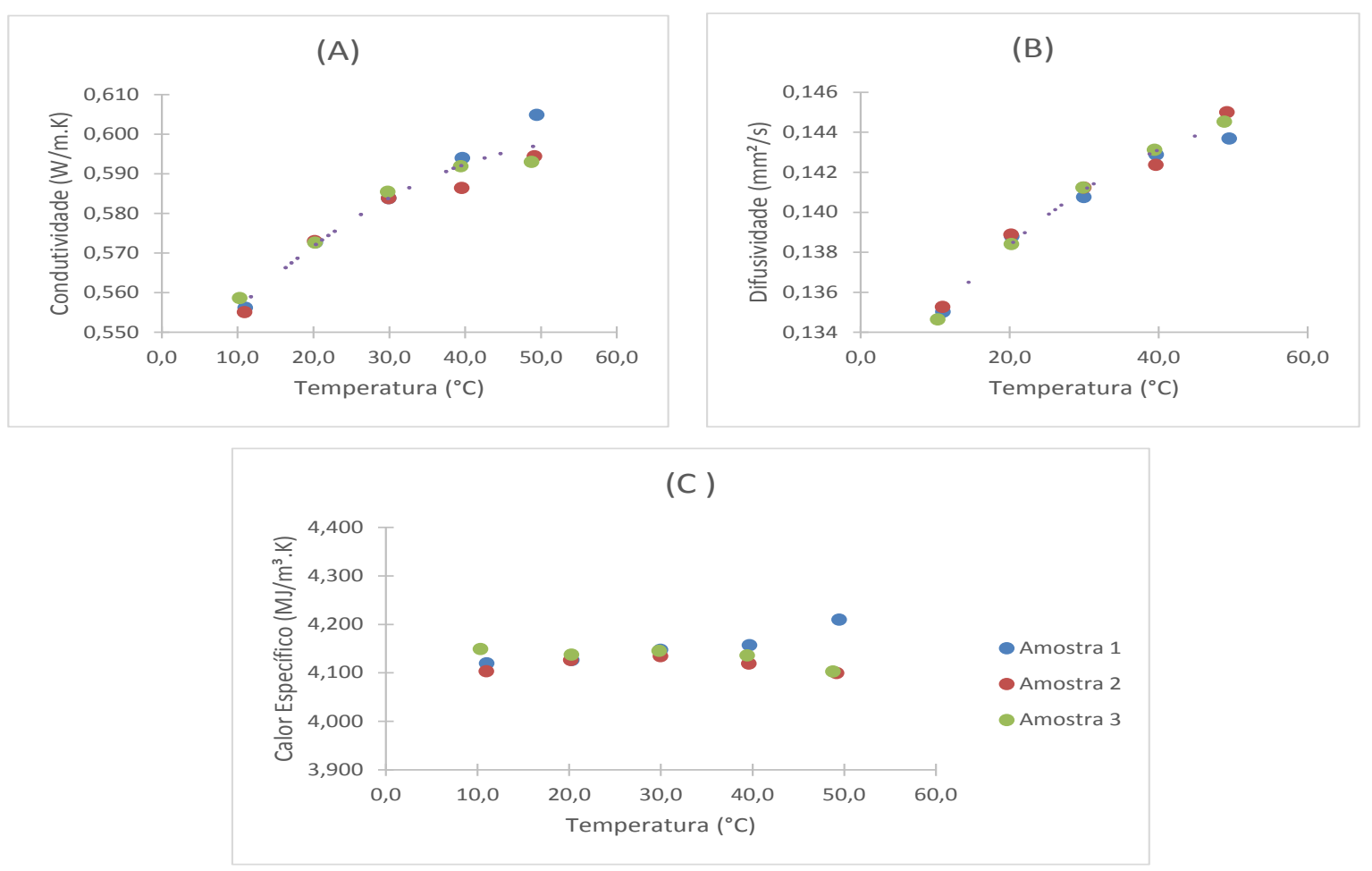

As correlações obtidas para a faixa de temperatura entre $10{ }^{\circ} \mathrm{C}$ e $50{ }^{\circ} \mathrm{C}$ para o suco de laranja foram as seguintes:

$$
\begin{array}{ll}
k=-1,83 \cdot 10^{-5} \cdot T^{2}+2,13 \cdot 10^{-3} \cdot T+5,36 \cdot 10^{-1} & \mathrm{R}^{2}=0,958 \\
\alpha=-3,86 \cdot 10^{-6} \cdot T^{2}+4,70 \cdot 10^{-4} \cdot T+1,31 \cdot 10^{-1} & \mathrm{R}^{2}=0,987
\end{array}
$$




$$
C p=4,13 \pm 0,03
$$

em que $k$ (condutividade térmica) é dado em $\mathrm{W} \mathrm{K}^{-1} \mathrm{~m}^{-1}, \alpha$ (difusividade térmica) em $\mathrm{mm}^{2} \mathrm{~s}^{-1}$, $C p$ (calor específico) em $\mathrm{MJ} \mathrm{m}^{-3} \mathrm{~K}^{-1}$ e $T$ em ${ }^{\circ} \mathrm{C}$. Não foi possível observar uma influência significativa da temperatura sobre o calor específico na faixa de temperatura testada. Entretanto, notou-se uma elevação da condutividade térmica com a temperatura, comportamento observado para a água, principal constituinte dos alimentos analisados. Consequentemente houve um aumento de comportamento similar na difusividade térmica, representado por um ajuste polinomial de segundo grau.

Da mesma maneira, foram preparadas três amostras de polpa de manga com ágar $(1,0 \%)$. Os valores obtidos na faixa de temperatura estudadas são apresentados na Figura 2.

Figura 2 - Gráficos de condutividade térmica (A), difusividade térmica (B) e calor específico volumétrico $(\mathrm{C})$ de polpa de manga.

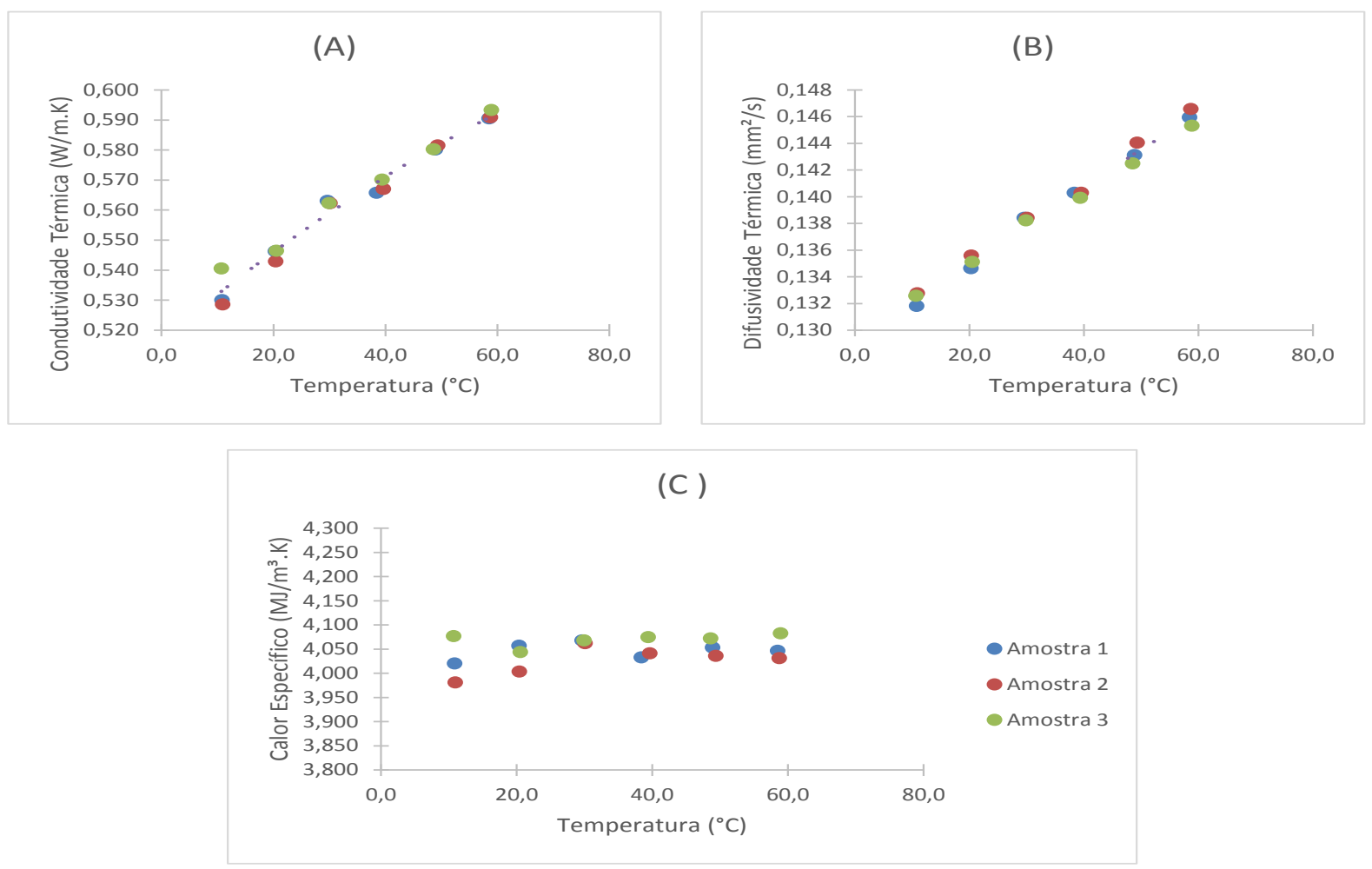

As correlações obtidas para a faixa de temperatura entre $10{ }^{\circ} \mathrm{C}$ e $60{ }^{\circ} \mathrm{C}$ para a polpa de manga foram as seguintes:

$$
\begin{array}{ll}
k=-4,92 \cdot 10^{-6} \cdot T^{2}+1,55 \cdot 10^{-3} \cdot T+5,17 \cdot 10^{-1} & \mathrm{R}^{2}=0,976 \\
\alpha=-2,58 \cdot 10^{-7} \cdot T^{2}+2,99 \cdot 10^{-4} \cdot T+1,29 \cdot 10^{-1} & \mathrm{R}^{2}=0,990 \\
C p=4,05 \pm 0,03 &
\end{array}
$$


em que $k$ é dado em $\mathrm{W} \mathrm{K}^{-1} \mathrm{~m}^{-1}, \alpha$ em $\mathrm{mm}^{2} \mathrm{~s}^{-1}, C p$ em $\mathrm{MJ} \mathrm{m}^{-3} \mathrm{~K}^{-1}$ e $T$ em ${ }^{\circ} \mathrm{C}$. Também observou-se um aumento da condutividade térmica com a temperatura e influência não significativa sobre o calor específico.

Em seguida, foi estudado o comportamento do suco de maçã, com preparo semelhante aos anteriores. Os resultados encontrados estão demonstrados na Figura 3.

Figura 3 - Gráficos de condutividade térmica (A), difusividade térmica (B) e calor específico volumétrico (C) do suco de maçã.

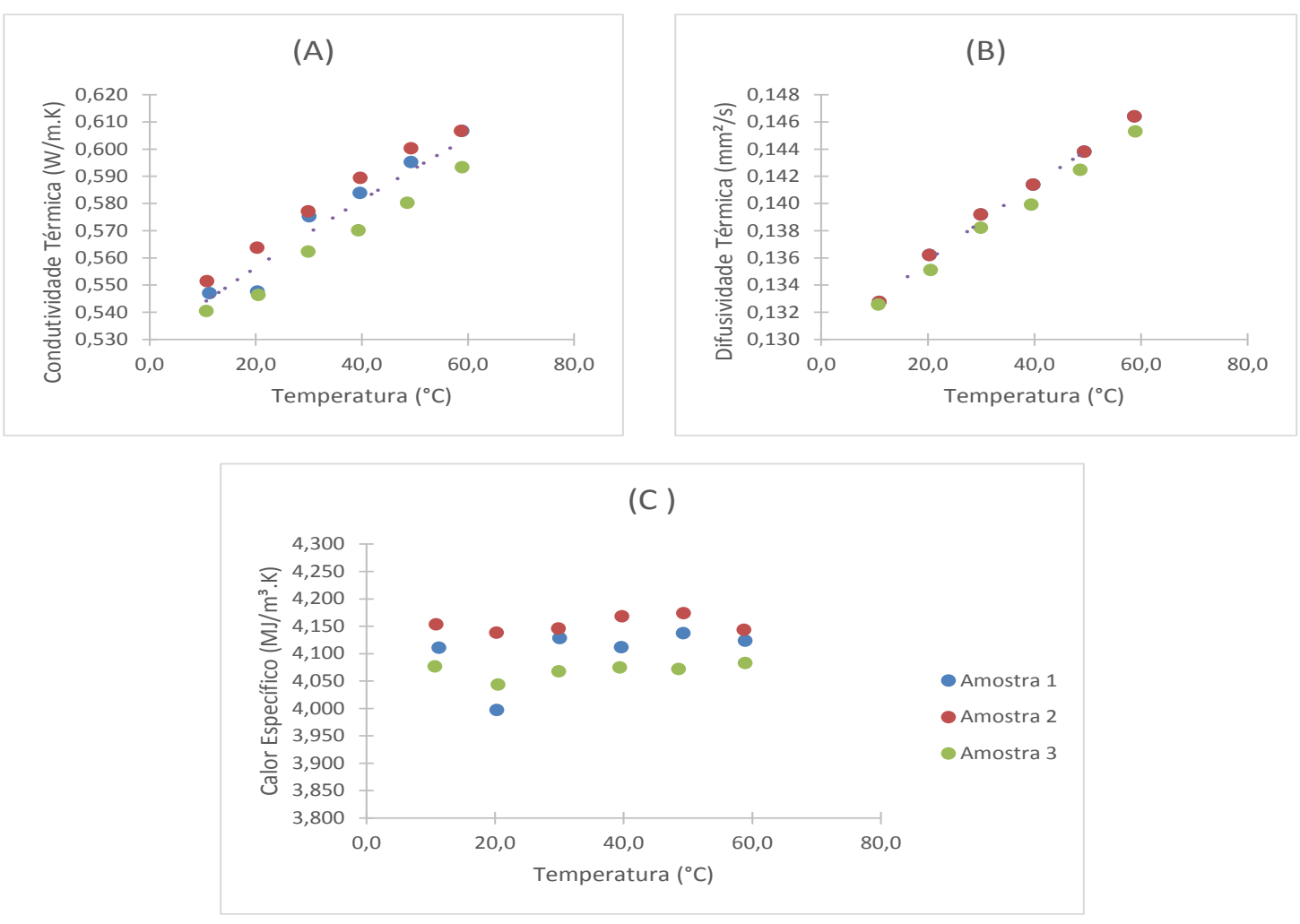

As correlações obtidas para a faixa de temperatura entre $10{ }^{\circ} \mathrm{C}$ e $60{ }^{\circ} \mathrm{C}$ para o suco de maçã foram as seguintes:

$$
\begin{array}{ll}
k=-2,55 \cdot 10^{-6} \cdot T^{2}+1,39 \cdot 10^{-3} \cdot T+5,30 \cdot 10^{-1} & \mathrm{R}^{2}=0,880 \\
\alpha=-8,50 \cdot 10^{-7} \cdot T^{2}+3,32 \cdot 10^{-4} \cdot T+1,29 \cdot 10^{-1} & \mathrm{R}^{2}=0,977 \\
C p=4,12 \pm 0,05 &
\end{array}
$$

em que $k$ é dado em $\mathrm{W} \mathrm{K}^{-1} \mathrm{~m}^{-1}, \alpha$ em $\mathrm{mm}^{2} \mathrm{~s}^{-1}, C p$ em $\mathrm{MJ} \mathrm{m}^{-3} \mathrm{~K}^{-1} \mathrm{e}^{T}$ em ${ }^{\circ} \mathrm{C}$.

Os três alimentos apresentaram propriedades térmicas em faixas próximas e com comportamento similar devido ao alto conteúdo de água. O método não foi eficaz para obter dados em temperaturas acima de $60{ }^{\circ} \mathrm{C}$, o que prejudica o uso dos resultados para cálculos de processos de pasteurização ou esterilização, por exemplo. 


\section{CONCLUSÕES}

Foi possível determinar as propriedades térmicas dos alimentos líquidos estudados pela técnica de sonda linear de aquecimento com imobilização por ágar; entretanto, o método não permitiu a determinação acima de $60{ }^{\circ} \mathrm{C}$, o que é uma limitação importante. Os resultados obtidos foram coerentes com o comportamento da água. Correlações polinomiais de ordens entre zero e dois foram ajustadas para modelar o efeito da temperatura, sendo que foi escolhido o menor número de parâmetros que fornecesse um ajuste satisfatório.

\section{REFERÊNCIAS}

DECAGON DEVICES, KD2 Pro Thermal Properties Analyzer, 4th ed., 2006.

HOOPER, F.G.; LEPPER, F.R. Transient heat flow apparatus for the determination of thermal conductivities, Process Biochemistry, 1975.

LIDE, D. R.; KEHIAIAN, H. V., CRC Handbook of Thermophysical and Thermochemical Data, CRC Press, 1994.

MURAKAMI, E.G.; SWEAT, V.E.; SASTRY, S.K.; KOLBE, E.; HAYAKAWA, K.; DATTA, A. Recommended design parameters for thermal conductivity probes for nonfrozen food materials, Journal of Food Engineering, 1996a.

MURAKAMI, E.G.; SWEAT, V.E., SASTRY, S.K., KOLBE, E. Analysis of various desing and operating parameters of the termal conductivity probe, Journal of Food Engeneering, 1996b.

NIX, G.H.; LOWERY, G.W.; VACHON, R.I.; TANGER, G.E. Direct determination of thermal diffusivity and conductivity with a refined line source technique, Progress in Aeronautics and Astronautics: Thermophysics of Spacecraft and Planetary Bodies, 1967.

RAHMAN, M. S. Food Properties Handbook, 2nd ed., CRC Press, 2009.

STALHANE, B.; PYK, S. New method for determining coefficients of thermal conductivity, Teknisk Tidskift, 1933

SUGAI, A.Y. Estudo do processamento contínuo de purê de manga (Mangifera indica Linn.), variedade Palmer. Tese (Doutorado). Escola Politécnica da USP, 2007.

SWEAT, V.E. Thermal Properties of Foods, Engineering Properties of Foods, 1986.

SWEAT, V.E.; HAUGH, C.G. A thermal conductivity probe for small food samples, Transactions of ASAE, 1974.

TRIBESS, T.B. Estudo da cinética de inativação térmica da pectinesterase em suco de laranja natural minimamente processado. Dissertação (Mestrado). Escola Politécnica da USP, 2003.

VAN DER HELD, E.F.M.; VAN DRUNEN, F.G. A method of measuring the thermal conductivity of liquids, Physica, 1949. 